\title{
Low Computational Cost Method to Calculate the Hosting Capacity in Radial Low Voltage Networks
}

Juan Morales-Conde, Sebastián Martín, Juan Pérez-Ruiz

Escuela de Ingenierías Industriales

Universidad de Málaga

Málaga, Spain

1st October 2019 


\section{Index}

(1) Introduction

3

(2) Methodology

7

(3) Case Study

19

(4) Conclusions and Summary

23 
Introduction 


\section{Introduction: Context}

- Prosumers (load + PV) $\rightarrow$ sizing, operation, management.

- Optimization problem.

- Voltage phasors and impedances $\rightarrow$ auxiliary variables.

- Comply with network constraints: overvoltage, ampacity. 


\section{Introduction: Literature Review}

S. M. Ismael et al, "State-of-the-art of hosting capacity in modern power systems with distributed generation," Renewable Energy, vol. 130, 2019

A) Convexification of network constraints:

- Sophisticated mathematics as second order cone programming.

$L$. Gan et al, "Exact convex relaxation of optimal power flow in radial networks," Automatic Control, IEEE Transactions on, vol. 60, Jan 2015

B) Hosting capacity (construction of feasible regions):

- Stochastic methods (sampling), as M. Rylander and J. Smith, "Stochastic Analysis to Determine Feeder Hosting Capacity for Distributed Solar PV," Tech. Rep. 1026640, EPRI, Dec. 2012

- Linearization of power flow equations:

M. Alturki et al, "Optimization-based distribution grid hosting capacity calculations," Applied Energy, vol. 219, 2018.

M. S. S. Abad et al, "Probabilistic assessment of hosting capacity in radial distribution systems," IEEE Transactions on Sustainable Energy, vol. 9, Oct 2018

Complex formulations and high computational cost. 


\section{Introduction: Proposed Method}

- Low computational cost.

- High accuracy.

- Use of Thévenin equivalent to map the high dimensional problem $\rightarrow$ low dimension space.

- Valid for low voltage radial networks.

- Allows for the construction of feasible operation regions.

- Comply with the network constraints in the optimization problem without including power flow equations. 
Methodology 


\section{Methodology: Problem for each user $k$}

- Difficulties:

- Uncertainty in some parameters (PV generation).

- Uncertainty on the other users actions (non-controllable loads).

- Simple loop circuit for each user $k$ :

- System Thévenin from user $k$ point of view.

- Thévenin of the user own installation.

- Each Thévenin parameter taking values in a certain region (contours of those regions are computed with the proposed method). 


\section{Methodology: Steps in the Method (I)}

1) Parameters for each user $k$ can take values in box type sets, $\left|S_{k}\right| \in\left[\left|\underline{S_{k}}\right|,\left|\overline{S_{k}}\right|\right], \ldots$

- Regions for the Thévenin parameters of the installation of each user $k$.

2) Regions for the Thévenin parameters of the whole system from the point of view of user $k$.

3) Simple loop circuit using the Thévenin parameters from the previous steps:

- Network constraints are applied on this circuit.

- Feasible regions for power injection of user $k$ are calculated. 


\section{Methodology: Steps in the Method (II)}
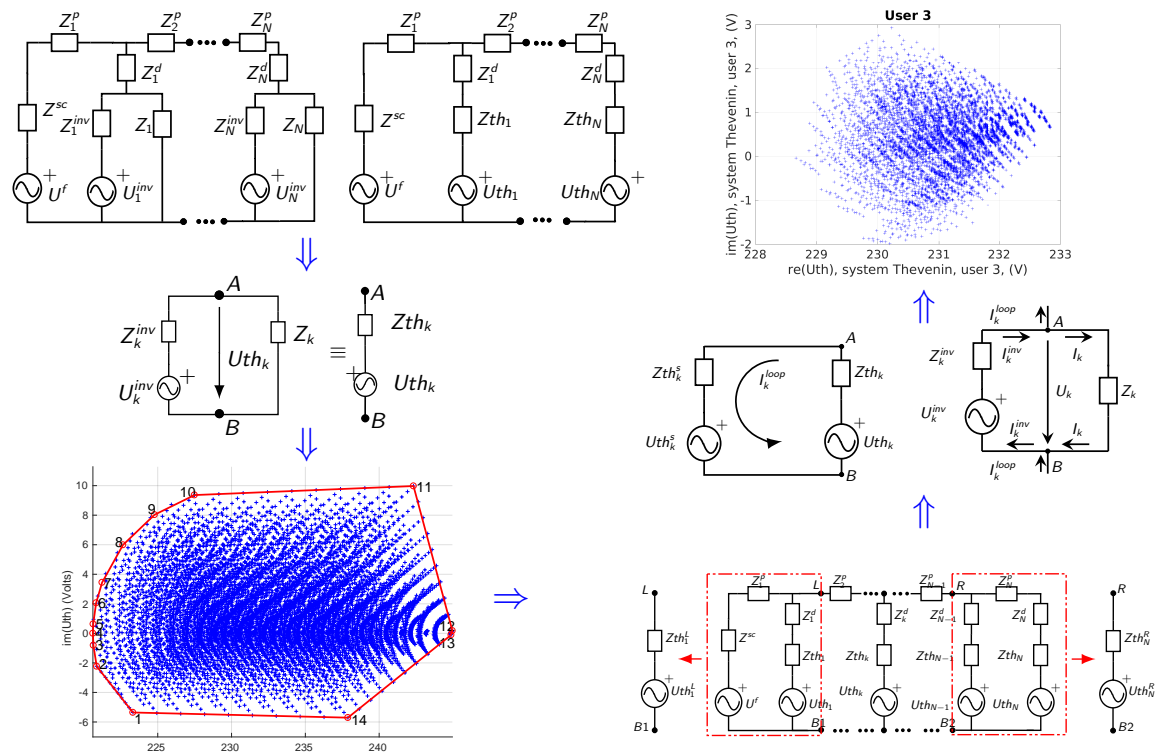


\section{Methodology: Thévenin for user k own installation} (I)

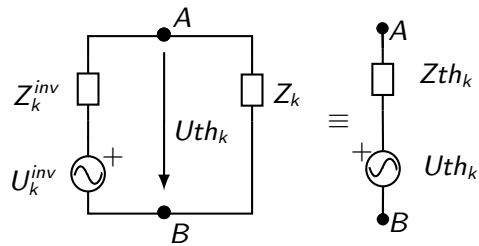

- User installation: load + PV

- Load of each user:

Equivalent impedance: $Z_{k}=\frac{\left|U^{\text {ref }}\right|^{2}}{\left|S_{k}\right|^{2}} S_{k}, \quad\left|S_{k}\right| \in\left[\left|\underline{S_{k}}\right|,\left|\overline{S_{k}}\right|\right]$

- PV installation:

- Ideal source of voltage: $U_{k}^{\text {inv }}$

- In series with impedance $Z_{k}^{i n v}=R_{k}^{i n v}+j \cdot X_{k}^{\text {inv }}$ $X_{k}^{i n v}=R_{k}^{i n v} \cdot \tan \left(\phi_{k}^{i n v}\right) ; R_{k}^{i n v}$ constant and $\phi_{k}^{i n v}$ variable 


\section{Methodology: Thévenin for user k own installation (II)}

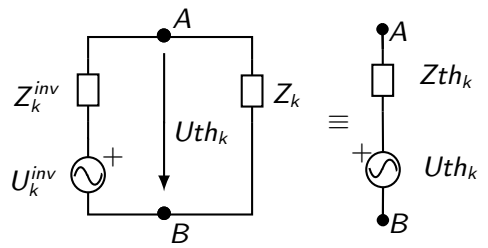

$Z t h_{k}$ and $U t h_{k}$ depend on four parameters: $\left|S_{k}\right|, \phi_{k},\left|U_{k}^{i n v}\right|$, and $\phi_{k}^{i n v}$.

$$
\begin{aligned}
Z t h_{k} & =\frac{Z_{k}^{i n v} \cdot Z_{k}}{Z_{k}^{i n v}+Z_{k}} \\
U t h_{k} & =U_{k}^{i n v} \cdot \frac{Z_{k}}{Z_{k}^{i n v}+Z_{k}}
\end{aligned}
$$




\section{Methodology: Region for $Z t h_{k}$}

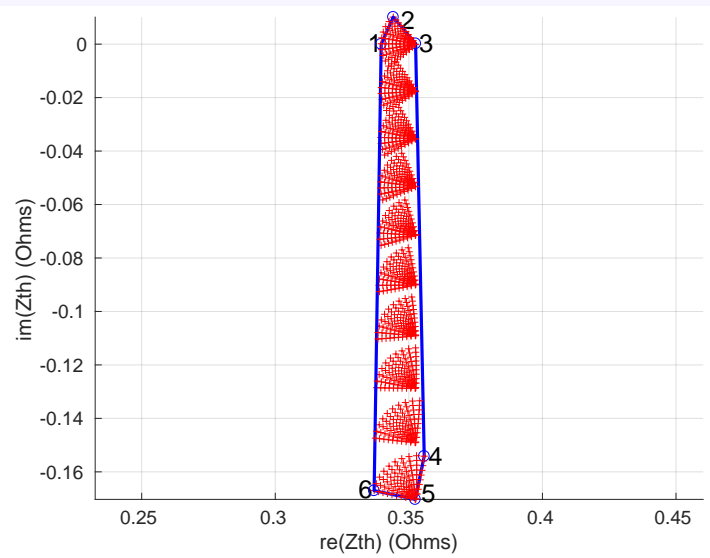

\begin{tabular}{c|llllll} 
Point & 1 & 2 & 3 & 4 & 5 & 6 \\
\hline$\phi_{k}^{i n v}$ & $\bar{\phi}_{k}^{i n v}$ & $\bar{\phi}_{k}^{i n v}$ & $\bar{\phi}_{k}^{i n v}$ & $\underline{\phi}_{k}^{i n v}$ & $\underline{\phi}_{k}^{i n v}$ & $\underline{\phi}_{k}^{i n v}$ \\
$\phi_{k}$ & 0 & $\bar{\phi}_{k}$ & $\bar{\phi}_{k}$ & $\bar{\phi}_{k}$ & $\bar{\phi}_{k}$ & $\frac{\phi}{k}$ \\
$\left|S_{k}\right|$ & $\left|\bar{S}_{k}\right|$ & $\left|\bar{S}_{k}\right|$ & $\left|\underline{S}_{k}\right|$ & $\left|\bar{S}_{k}\right|$ & $\left|\underline{S}_{k}\right|$ & $\left|{ }^{\prime}\right|$
\end{tabular}




\section{Methodology: Region for Uth}

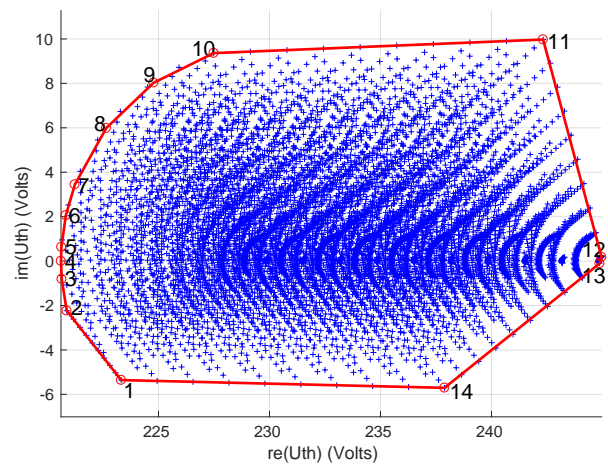

\begin{tabular}{|c|c|c|c|c|}
\hline Point & $\phi_{k}^{i n v}$ & $\phi_{k}$ & $\left|S_{k}\right|$ & $\left|U_{k}^{i n v}\right|$ \\
\hline 1 & $\bar{\phi}_{k}^{i n v}$ & $\phi_{k}$ & $\left|\bar{S}_{k}\right|$ & $\left|\underline{U}_{k}^{i n v}\right|$ \\
\hline 2 & $\phi_{k}^{i n v}$ & $\bar{\phi}_{k}^{n}$ & $\left|\bar{S}_{k}\right|$ & $\left|\underline{U}_{k}^{i n v}\right|$ \\
\hline 3 & $\phi_{k}^{i n v}$ & $\phi_{k}+0.1 \cdot\left(\bar{\phi}_{k}-\phi_{k}\right)$ & $\left|\bar{S}_{k}\right|$ & $\left|\underline{U}_{k}^{i n v}\right|$ \\
\hline 4 & $\underline{\phi}_{k}^{i n v}$ & $\underline{\phi}_{k}^{i n v}$ & $\left|\bar{S}_{k}\right|$ & $\left|\underline{U}_{k}^{i n v}\right|$ \\
\hline 5 & $\underline{\phi}_{k}^{i n v}$ & $\underline{\phi}_{k}+0.2^{-k} \cdot\left(\bar{\phi}_{k}-\underline{\phi}_{k}\right)$ & $\left|\bar{S}_{k}\right|$ & $\left|\underline{U}_{k}^{i n v}\right|$ \\
\hline 6 & $\phi_{k}^{i n v}$ & $\bar{\phi}_{k}^{k}+0.3 \cdot\left(\bar{\phi}_{k}-\bar{\phi}_{k}\right)$ & $\left|\bar{S}_{k}\right|$ & $\left|\underline{U}_{k}^{i n v}\right|$ \\
\hline 7 & $\phi_{k}^{i n v}$ & $\bar{\phi}_{k}^{n}+0.4 \cdot\left(\bar{\phi}_{k}-\bar{\phi}_{k}^{n}\right)$ & $\left|\bar{S}_{k}\right|$ & $\left|\underline{U}_{k}^{i n v}\right|$ \\
\hline 8 & $\underline{\phi}_{k}^{i n v}$ & $\underline{\phi}_{k}+0.6 \cdot\left(\bar{\phi}_{k}-\underline{\phi}_{k}\right)$ & $\left|\bar{S}_{k}\right|$ & $\left|\underline{U}_{k}^{i n v}\right|$ \\
\hline 9 & $\underline{\phi}_{k}^{i n v}$ & $\bar{\phi}_{k}^{n}+0.8 \cdot\left(\bar{\phi}_{k}-\bar{\phi}_{k}^{n}\right)$ & $\left|\bar{S}_{k}\right|$ & $\left|\underline{U}_{k}^{i n v}\right|$ \\
\hline 10 & $\underline{\phi}_{k}^{i n v}$ & ${ }^{-\kappa} \quad \bar{\phi}_{k}$ & $\left|\bar{S}_{k}\right|$ & $\left|\underline{U}_{k}^{i n v}\right|$ \\
\hline 11 & $\phi_{k}^{i n v}$ & $\bar{\phi}_{k}$ & $\left|\bar{S}_{k}\right|$ & $\left|\bar{U}_{k}^{i n v}\right|$ \\
\hline 12 & $\underline{\phi}_{k}^{i n v}$ & $\bar{\phi}_{k}$ & $\left|\underline{S}_{k}\right|$ & $\left|\bar{U}_{k}^{i n v}\right|$ \\
\hline 13 & $\overline{\bar{\phi}}_{k}^{i n v}$ & $\underline{\phi}_{k}$ & $\left|\underline{S}_{k}\right|$ & $\left|\bar{U}_{k}^{i n v}\right|$ \\
\hline 14 & $\bar{\phi}_{k}^{i n v}$ & $\underline{\phi}_{k}$ & $\left|\bar{S}_{k}\right|$ & $\left|\bar{U}_{k}^{i n v}\right|$ \\
\hline
\end{tabular}




\section{Methodology: Equivalent system from user $k$ point of view (I)}

- $Z t h_{k}^{s}, U t h_{k}^{s}$ Thévenin parameters for the system, point of view of user $k$.

- For a system with $N$ users, the regions for $Z t h_{k}^{s}, U t h_{k}^{s}$ are computed in $N-1$ steps.

- Based on observation from numerical experiments (no formal proof):

1) Only the regions for the equivalent of two parallel branches are computed at each step.

2) Calculation based on the points in the contours of the regions.

3) Regions for the new branch parameters are defined by their contour. 


\section{Methodology: Equivalent system from user $k$ point of view (II)}

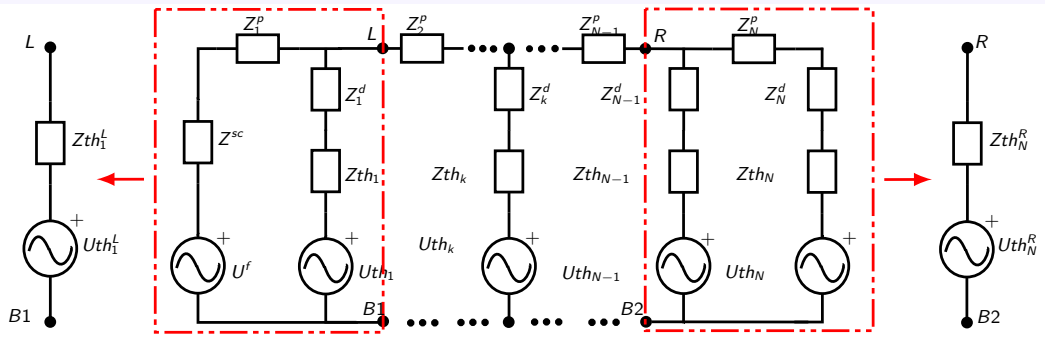

- Let be a system with $N$ users, and $M$ sample points for each parameter (four parameters per user).

- Less than 30 points needed to be checked to define a contour (observed).

- Computational cost (for 11 users and 10 points for parameter, $N=11$, $M=10)$ :

1) Proposed method $\approx(N-1) \cdot 30 \cdot 30 \Rightarrow 9000$.

2) Sampling method $M^{4 \cdot N} \Rightarrow 10^{44}$. 


\section{Methodology: Results for each user $k$}
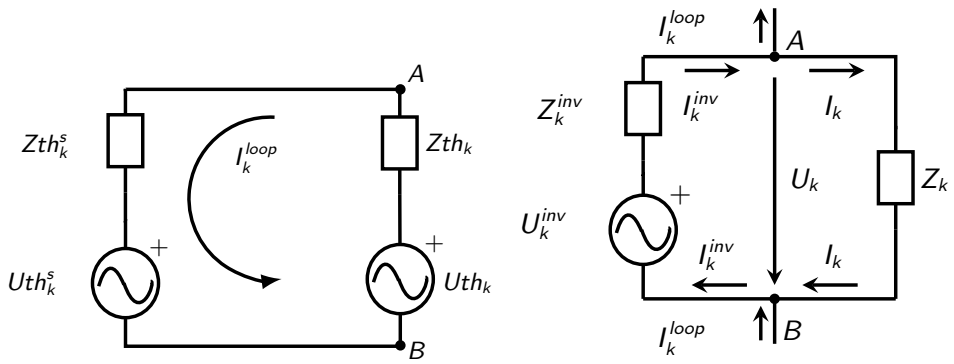

Unknowns: $I_{k}^{\text {loop }}, U_{k}, I_{k}$

$I_{k}^{\text {loop }}=\frac{U t h_{k}-U t h_{k}^{s}}{Z t h_{k}^{s}+Z t h_{k}}$

$U_{k}=U t h_{k}-l_{k}^{\text {loop }} \cdot Z t h_{k}$

$I_{k}=\frac{U_{k}}{Z_{k}}$

$$
I_{k}^{i n v}=I_{k}+I_{k}^{\text {loop }}
$$

(4) $U_{k}^{i n v}=Z_{k}^{i n v} \cdot I_{k}^{i n v}+U_{k}$

(5) $P_{k}^{i n v}=\operatorname{Re}\left[U_{k}^{i n v} \cdot\left(I_{k}^{i n v}\right)^{*}\right]$ 


\section{Methodology: Recommendations to comply with network constraints}

- Inverter efficiency $R_{k}^{i n v}$ strongly linked to load voltage $U_{k}$.

- Inverter impedance angle $\phi_{k}^{i n v}$ linked to: $I_{k}^{i n v}, I_{k}, I_{k}^{\text {loop }}$.

- System Thévenin impedance $Z t h_{k}^{s}$ strongly linked to: $\left|I_{k}^{\text {loop }}\right|,\left|U_{k}^{i n v}\right|$.

- System Thévenin impedance $Z t h_{k}^{s}$ weakly linked to: $Z_{k}$ (load impedance). 


\section{Case Study}




\section{Case Study: Example with three users}

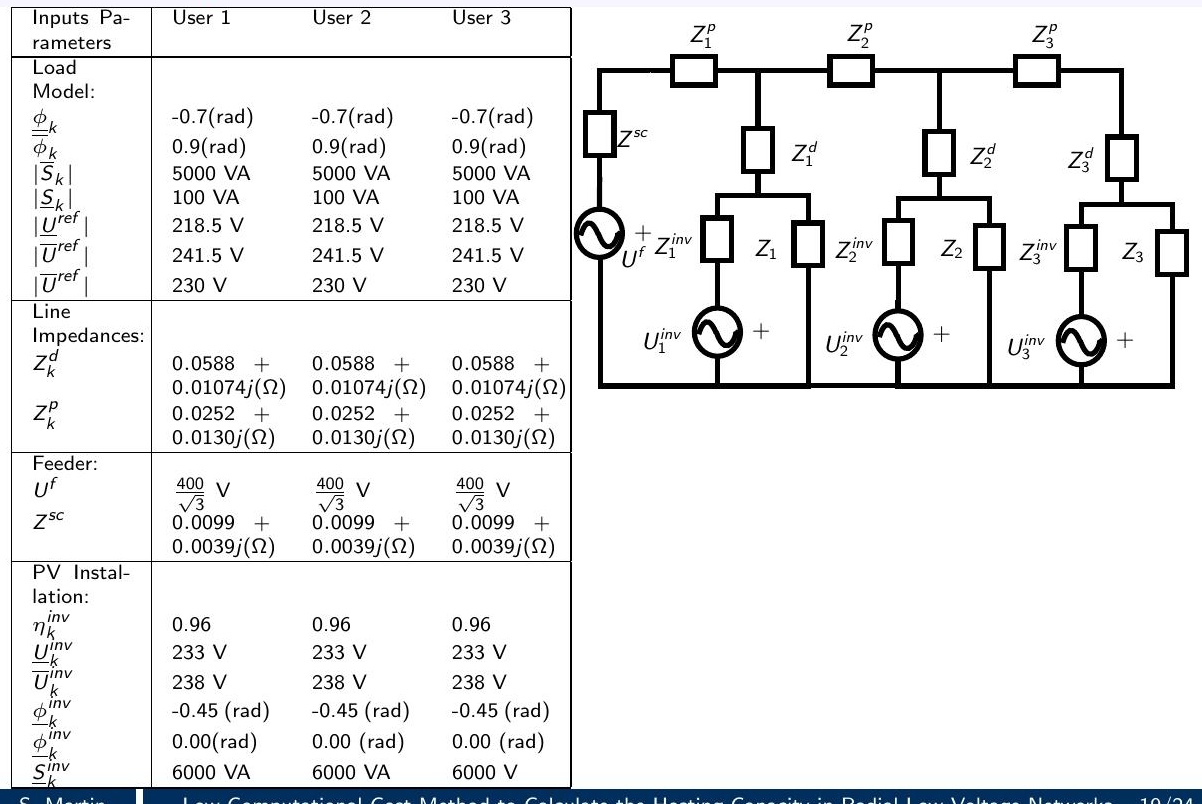




\section{Case Study: Results (I)}

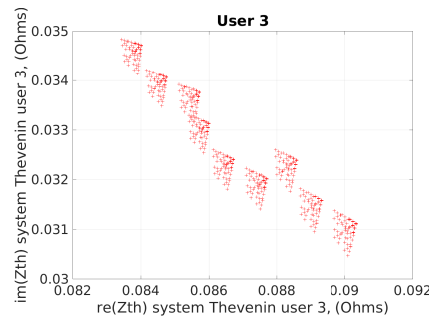

(g)

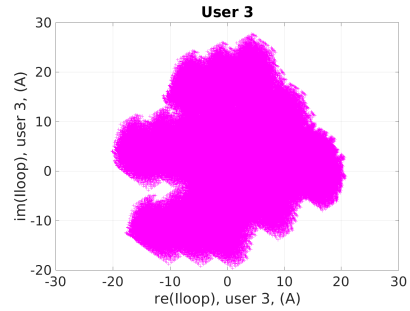

(i)

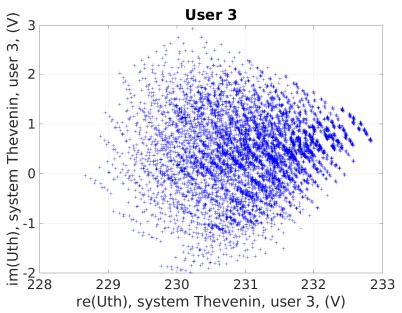

(h)

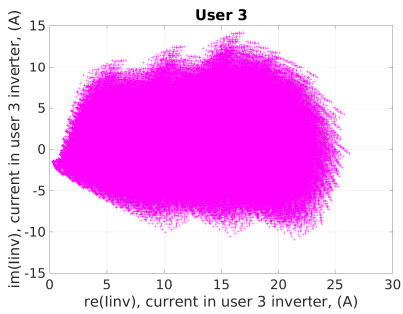

(j) 


\section{Case Study: Results (II)}

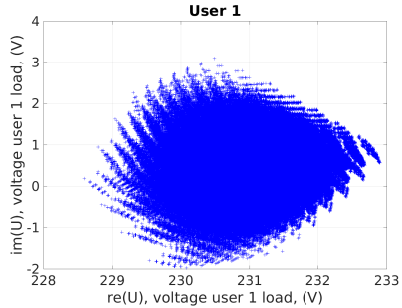

(k)

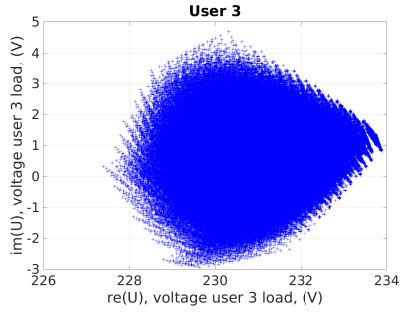

(m)

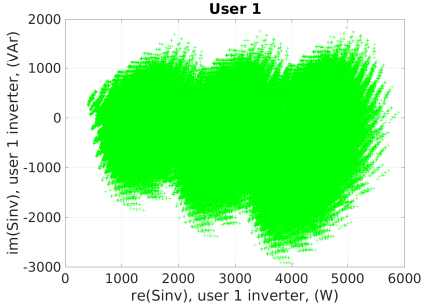

(I)

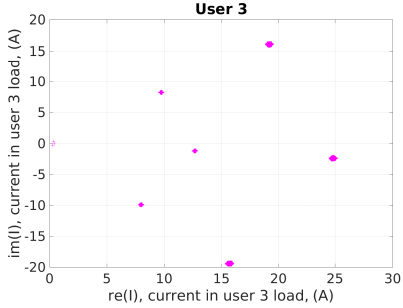

(n) 


\section{Conclusions and Summary}




\section{Conclusions and Summary}

- Thévenin mapping, from high dimension to low dimension space.

- Parameters regions defined by their contour in the Thévenin mapping.

- Low computational cost.

- It can be applied to a system with large number of users and/or repetitive processes:

- Calculation of feasible operation for each user.

- Optimal allocation of distributed generation in a low voltage radial network.

- Calculation of hosting capacity for each user.

- Based on observed results from numerical experiments.

- Currently working on formal proofs for the observed results. 
Thanks for your attention! 